\title{
Herzlich willkommen in Erfurt!
}

W ir freuen uns sehr, dass Sie unserer Einladung zum Dritten Deutschen Allergiekongress nach Erfurt gefolgt sind! Sie finden in dieser Sonderausgabe von Allergo Journal die Abstracts der eingereichten wissenschaftlichen Beiträge.

Der wissenschaftliche Beirat hat ein Programm zusammengestellt, das sich an den Bedürfnissen des klinisch und wissenschaftlich arbeitenden Allergologen orientiert. Die Themenauswahl ist darauf ausgerichtet, die allergologische Tätigkeit zu unterstützen. Dazu wurde auf Themen Wert gelegt, die das Verständnis für Zusammenhänge zwischen Pathogenese, klinischem Verlauf, diagnostischen Maßnahmen, Therapieoptionen und Versorgungsmöglichkeiten im deutschen Gesundheitswesen fördern.

Vor der feierlichen Eröffnung des Kongresses findet am Mittwoch von 16.30 bis 19.00 Uhr das internationale Joint Symposium von DGAKI und EAACI Immunology Section statt. Hochkarätige Referenten aus Europa und Australien werden aktuellste Forschungsergebnisse präsentieren. Als Special Guest des Kongresses wird Prof. Susan Prescott aus Perth zum Vortrag „Allergy Prevention" am Samstagvormittag erwartet.

Zentrale Punkte des Programms sind die Plenarsitzungen mit Übersichtsvorträgen, die der Pathogenese und Klinik

„Auf vielfältige Anregung hin werden Fortbildungskurse angeboten, um nach der Theorie der Plenar- und Symposiumssitzungen die Handlungsanleitungen für den klinischen Alltag zu vertiefen.“ schwerer allergischer Erkrankungen, nicht IgE-vermittelten allergischen Erkrankungen und Neuem zur kausalen Therapie der Allergologen, der allergenspezifischen Immuntherapie, gewidmet sind. In den nachfolgenden, parallel organisierten Symposien werden weitere Themen aufgenommen und präsentiert. Eine Übersicht dazu finden Sie auf Seite S102.

Ein berufspolitisches Symposium soll den Praktikern Hilfe bei der Bewältigung des kassenärztlich-allergologischen, sich stark verändernden Alltags bieten. Die chronische Unterfinanzierung des Gesundheitssystems führt seit 2004 zu einer kontinuierlichen Verschlechterung der Situation der Allergiekranken. Hier kommen auch die $\mathrm{Pa}-$ tienten zu Wort mit einem Referat von Ingrid Voigtmann vom Deutschen Allergie- und Asthmabund. Dr. Bernhard Rochell von der Honorarabteilung der Kassenärztlichen Bundesverei-

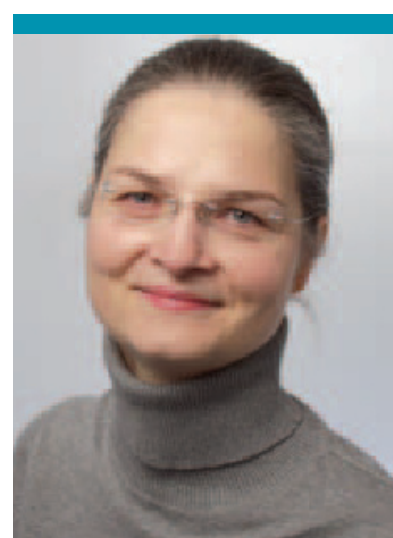

Priv.-Doz. Dr. Kirsten Jung, Praxis für Dermatologie und Immunologie, Erfurt

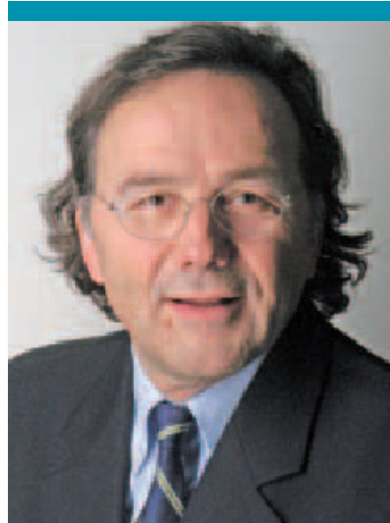

Prof. Dr. Thomas Fuchs, Abteilung Dermatologie und Venerologie, Universitätsmedizin Göttingen, GeorgAugust-Universität, Göttingen nigung hat sein Kommen zum Thema Zukunft des allergologischen Labors beim klinisch tätigen Facharzt zugesagt. Dies wird die Allergologen aufgrund der beschlossenen Sparmaßnahmen zur allergologischen In-vitro-Diagnostik ab 1. Januar 2009 besonders interessieren.

Der Allergiekongress hat wiederum dem wissenschaftlichen Nachwuchs besondere Präsentationsmöglichkeiten eingeräumt. Neben den Symposien für freie Vorträge finden fünf Sitzungen mit Posterdiskussionen statt.

Auf vielfältige Anregung hin werden Fortbildungskurse angeboten, um nach der Theorie der Plenar- und Symposiumssitzungen am Samstagnachmittag die Handlungsanleitungen für den klinischen Alltag zu vertiefen. Hierzu gibt es sowohl für Ärzte als auch für das mittlere medizinische Personal zahlreiche Angebote.

Nach den wissenschaftlichen Tagesprogrammen wird das Orgelkonzert im Dom am Donnerstag und der Gesellschaftsabend im Kaisersaal am Freitag, dem Ort des von Napoleon vor 200 Jahren einberufenen Fürstenkongresses, neben Spaziergängen durch den mittelalterlichen Stadtkern Möglichkeiten für Erholung und Fortsetzung der kollegialen Gespräche bieten.
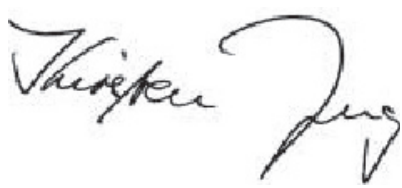

Priv.-Doz. Dr. Kirsten Jung, Kongresspräsidentin

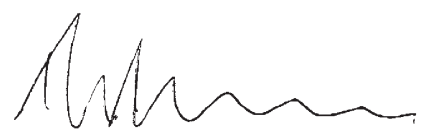

Prof. Dr. Thomas Fuchs, Kongresspräsident 